Assessing physical activity intensity by video analysis

This content has been downloaded from IOPscience. Please scroll down to see the full text. 2015 Physiol. Meas. 361037

(http://iopscience.iop.org/0967-3334/36/5/1037)

View the table of contents for this issue, or go to the journal homepage for more

Download details:

IP Address: 193.136.33.149

This content was downloaded on 12/06/2015 at 17:54

Please note that terms and conditions apply. 


\title{
Assessing physical activity intensity by video analysis
}

\author{
P Silva ${ }^{1}$, C Santiago ${ }^{2}$, L P Reis ${ }^{3}$, A Sousa $^{2}, \mathrm{~J} \mathrm{Mota}^{1}$ and \\ G Welk \\ ${ }^{1}$ CIAFEL, Faculty of Sports, University of Porto, Portugal \\ 2 INESC TEC and Faculty of Engineering, University of Porto, Portugal \\ 3 School of Engineering, University of Minho and LIACC, Faculty of Engineering, \\ University of Porto, Portugal \\ ${ }^{4}$ Dept. of Kinesiology, Iowa State University, Ames, IA 50011, USA \\ E-mail: perrinha@gmail.com,pro09005@fe.up.pt, lpreis@dsi.uminho.pt, asousa@ \\ fe.up.pt, jmota@fade.up.pt and gwelk@iastate.edu
}

Received 10 November 2014, revised 8 March 2015

Accepted for publication 11 March 2015

Published 22 April 2015

\begin{abstract}
Assessing physical activity (PA) is a challenging task and many different approaches have been proposed. Direct observation (DO) techniques can objectively code both the behavior and the context in which it occurred, however, they have significant limitations such as the cost and burden associated with collecting and processing data. Therefore, this study evaluated the utility of an automated video analysis system (CAM) designed to record and discriminate the intensity of PA using a subject tracking methodology. The relative utility of the CAM system and DO were compared with criterion data from an objective accelerometry-based device (Actigraph GT3X+). Eight 10 year old children (three girls and five boys) wore the GT3X+ during a standard basketball session. PA was analyzed by two observers using the SOPLAY instrument and by the CAM system. The GT3X+ and the CAM were both set up to collect data at $30 \mathrm{~Hz}$ while the DO was performed every two minutes, with $10 \mathrm{~s}$ of observation for each gender. The GT3X+ was processed using cut points by Evanson and the outcome measure was the percentage of time spent in different intensities of PA. The CAM data were processed similarly using the same speed thresholds as were used in establishing the Evenson cut-off points (light: <2 mph; walking: 2-4 mph; very active: $>4 \mathrm{mph}$ ). Similar outcomes were computed from the SOPLAY default analyses. A chi-square test was used to test differences in the percentage of time at the three intensity zones (light, walking and very active). The Yates' correction was used to prevent overestimation of statistical significance for small data. When compared with GT3X+, the CAM had better results than the SOPLAY. The chi-square test
\end{abstract}


yielded the following pairwise comparisons: CAM versus GT3x+ was $\chi^{2}(5)$ $=24.18, p<.001$; SOPLAY2 versus GT3x+ was $\chi^{2}(5)=144.44, p<.001$; SOPLAY1 versus GT3x+ was $\chi^{2}(5)=119.55, p<.001$. The differences were smaller between CAM and GT3x+, suggesting that the video tracking system provided better agreement than DO. The small sample size precludes a definitive evaluation but the results show that the CAM video system may have promise for automated coding of physical activity behavior.

Keywords: motion classification, accelerometer, direct observation, video tracking

(Some figures may appear in colour only in the online journal)

\section{Introduction}

Assessing physical activity (PA) is a challenging task and many different approaches have been proposed. Accelerometry based activity monitors have gained acceptance as a standard for field based research. These objective measures with real time data storage capabilities offer distinct advantages: they provide reliable information on patterns of PA over several days (Trost et al 2000); they can be programmed to start and stop recording data at a desired time period, which can distinguish different time contexts, such as school time, after-school time, weekends and so on (Mota et al 2008, Silva et al 2011, Saint-Maurice et al 2011b). Accelerometers are a relevant tool because they describe the workings of PA in a very detailed way, and their global acceptance allows them to be used in large studies (Riddoch et al 2004, Hagstromer et al 2007, Troiano et al 2008). While these devices are widely used and accepted, there are challenges that must be overcome to advance research on PA behavior. The main one is the lack of contextual information provided about physical activity. To try and overcome this challenge, accelerometers can be combined with qualitative information, such as the space and settings variables where PA is realized, and this way it will significantly improve the information necessary for PA promotion and guidelines.

Research has been undertaken to try to describe in more detail PA behavior, and a wide range of different instruments have been used for this, such as daily logs, diaries, questionnaires, direct observation, GPS (global positioning system), GIS (geographical information system), cell phones, and EMA (electronic ecological momentary assessment). Concurrently, advanced technologies and modeling techniques have also led to the development of new pattern recognition algorithms that provide alternative ways of monitoring and evaluating PA. Today there are devices available to the general public that can be used in the research context.

The direct observation (DO) method is often viewed as the most effective (gold standard) technique for youth related research because behavior is directly observed (McKenzie 2010). There are several different instruments that use observation techniques to generate PA data, a detailed description of them is not within the scope of this manuscript, but the more commonly used are: BEACHES — behaviors of eating and physical activity for children's health; CARS—children's activity rating scale; SOFIT—System for observing fitness instructor time; SOPLAY — system for observing play and leisure activity in youth; and SOPARC—-system for observing play and recreation in communities.

The most widely used DO tool is the SOFIT and this tool has been validated using a variety of methods (McKenzie 2002). A limitation of SOFIT is that it can only be used to evaluate activity behaviors of one individual at a time. Hence, the cost and burden limit its utility for 
field based research. To address this limitation, an alternative tool called SOPLAY was developed. This instrument was specifically designed to facilitate the observation of groups and environmental contexts (McKenzie et al 2000). The SOPLAY assesses PA levels and contextual factors using momentary group time sampling techniques. However, a limitation of this method is that it does not provide insights into the individual variability in behaviors within a group (Saint-Maurice et al 2011b). A more significant limitation of SOPLAY (and all other DO methods) is the inherent burden of capturing, coding and processing data.

Hence, research is warranted to explore the potential of automatic observation tools that can address the limitations of the current DO techniques. By using a video recording system, researchers can capture both the context and the behavior of more than one individual at once. Advanced video-based tracking systems have been shown to have considerable promise in surveillance and security applications. There are also several applications currently used in professional sports, such as football and basketball, and these same techniques can be applied to enhance the monitoring of PA. Video has the advantage of being non-intrusive, continuous, and permanent (behavior is recorded without interruption and can be reviewed over time). In this study, we evaluate the utility of an innovative video tracking system designed to capture and discriminate PA intensities. The video observation (conducted during a basketball practice in an indoor gymnasium) was compared with standard SOPLAY DO methods and accelerometry based monitors (criterion measure) to evaluate convergent validity. We hypothesized that the video method would capture PA intensities as effectively as the more established SOPLAY method.

\section{Methods}

The video recording and tracking system (CAM) was installed in a sports club, and participants were observed during a basketball practice. Parents and the coach provided consent prior to the beginning of the study and the study protocol was explained in detail to the participants. A total of eight 10 year old participants (three girls and five boys), wore the Actigraph GT3X+ during a $20 \mathrm{~min}$ regular basketball session. The only constraint on the practice session was for participants to remain inside the surveyed area (i.e. the entire basketball court). Observations were made simultaneously during the entire regular practice session (coach talk, warm-up, skill circuit and a full-court game) with both the SOPLAY instrument (two observers) and the automated CAM system.

\subsection{Accelerometer data}

ActiGraph GT3X+ accelerometers (ActiGraph; Pensacola, FL, USA) were used to assess the PA intensity of each participant. With the help of the research team, the accelerometers were fixed to each child's waist with an adjustable elastic belt over the right iliac crest, prior to the start of the observed practice. Monitors were initialized to be synchronized with the video recording system to ensure temporal links with the data. The GT3X+ was set to collect data in three axes of motion, in raw mode at $30 \mathrm{~Hz}$. Data were processed with the Actilife software (version 6.3) and the raw accelerometer counts were saved to a customized Microsoft Excel file. All of the GT3x+ data were filtered and processed to extract the specific activity during serial $10 \mathrm{~s}$ observations periods (designed to match the data obtained with the SOPLAY). The percentages of time spent in different PA intensities were determined using cut points proposed by Evenson et al (Evenson et al 2008). Since these cut points were derived from the vertical axis, in this study we also only used the vertical axis data. Our decision to use the 
Evenson work to calibrate our accelerometer data is supported by the fact that the age of the participants and the activities were similar to those tested in the calibration study (i.e. basketball, walking and running). To provide a more appropriate comparison with the DO and CAM system, we used the established GT3X+ cut points to compute the percentage of time spent in three different intensity zones (light: $<2 \mathrm{mph}$; walking: $2-4 \mathrm{mph}$; very active: $>4 \mathrm{mph}$ ).

\subsection{Direct observation-SOPLAY}

SOPLAY is designed to assess group levels of physical activity in different settings and environmental contexts (McKenzie 2002). It uses momentary group time sampling to code PA in three categories: sedentary, walking and very active (McKenzie et al 2006). SOPLAY data in this study followed the standard protocol with data collected separately for boys and girls in a pre-determined target area (i.e. mini-basketball court). Each individual's physical activity was scanned and coded by two observers every two minutes, with $10 \mathrm{~s}$ of observation for each gender. This frequency was chosen based on previous research (Saint-Maurice et al 2011a) demonstrating that more frequent scans can improve the validity of the estimations. The two observers were synchronized with the same clock, so that each scan was done at the exact same time, starting from the left to the right of the target zone, scanning one team first and the other afterwards. DO data were annotated by hand in the proper SOPLAY form and transcribed later to a customized Microsoft Excel file. The data were processed to compute the percentage of time children spent in the three different activity categories.

\subsection{The video recording system (CAM)}

A non-invasive, automatic video tracking system (CAM), composed of one video camera for detecting and tracking players was used (figure 1). Dedicated camera systems are mostly used in indoor environments because the smaller playing area makes it possible to use a single camera (Needham and Boyle 2001). The ceiling was chosen as the best spot to set the camera system, since there is no interference from the crowd, a single player never fills the entire field of view of the camera and a bird's eye perspective usually means less occlusion and/or merging situations (this solution was also adopted by (Kristan et al 2009, Monier et al 2009). The proposed system uses the Gigabit Ethernet camera DFK 31BG03.H model from Imaging Source. Resolution is $1024 \times 768$ pixels and the camera delivers 30 frames per second. The lenses used are Computar T2Z1816CS varifocal lenses with focal distances ranging from 1.8 to $3.6 \mathrm{~mm}$ (wide angle lens). The choice of industrial grade gigabit ethernet interfaced cameras brings reliability with digital quality, high data rate and low cost. It uses common hardware and low cable costs while still capturing action across large distances.

Advanced image and video processing techniques were used and involved three steps: image segmentation; player detection; and player tracking (Santiago et al 2012). A three module software system was used: one responsible for acquiring the images from the camera (Acquisition System); the second for the off-line processing of the video stream (Processing System) that detects and tracks the players and generates a log file with the players' positions; and the third module (Visualizer) is able to merge the video stream and the log file to create a global image of the field with the players highlighted, so that the user can later analyze the collected information (figure 2). The use of real-world coordinates allows transparent tracking - when a user selects a player to be tracked, a new Kalman filter is added to the vector with the player's real world position and a default velocity of $0 \mathrm{~m} \mathrm{~s}^{-1}$. Afterwards, the players' locations on the subsequent frames are predicted. For the cases when the tracking is 

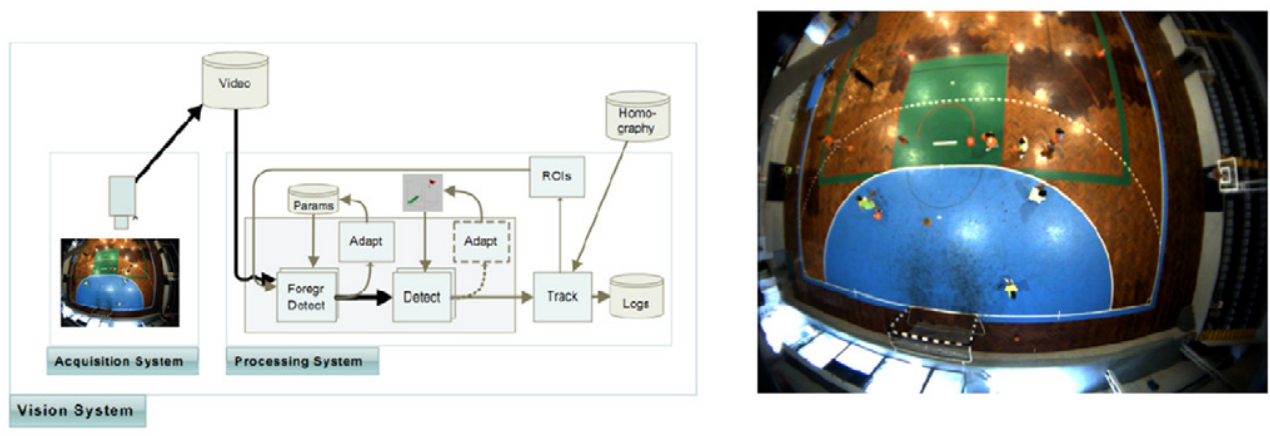

Figure 1. CAM system setup and software.

lost beyond a given configurable threshold (called the tracking prediction window, TPW), the system prompts the user to manually locate the player. If subsequent tracking is successful, no further user actions are required and current tracking is linked to previous history as a normal result of the Kalman filter.

The resulting log file, generated during the processing of the frames, contains the players' positions and velocities. The Visualizer can use this file not only to see the images with the tracked players highlighted, but also to extract measurements of the players' behaviors. The methodology adopted is the same used in a previous study done in handball by (Santiago et al 2012). To perform this analysis in an automated, formal and accurate way, these authors developed a cost conscious processing system that detected players by vest colors, and their tracking was enhanced using Kalman filtering. By using simple features such as color combined with a powerful tool for tracking (Kalman filter), it is possible to detect and track players throughout the game area with very limited user intervention; initial color calibration by user and little more. The typical processing time of the CAM system is about $160 \mathrm{~ms}$ to process each frame using an Intel Core i7-2630QM@ (2.00-2.90 GHz) computer operating on Windows7. Since the player's velocity is not constant throughout the game, at each frame each player's velocity is updated and classified into different intensity categories. The speed thresholds used for the segmentation (i.e. light: $<2 \mathrm{mph}$; walking: $2-4 \mathrm{mph}$; very active: $>4 \mathrm{mph}$ ) are the same ones used by Evenson et al to establish the accelerometer cut points in their study (Evenson et al 2008). By predicting the position of the players on the subsequent frames it is possible to reduce the computational cost because only a few regions of the entire image are searched for players. Despite some misdetections, the tracking achieved very good rates, having a success that ranged from 95.44 to $99.90 \%$ (and a corresponding average of $98.79 \%$ ).

\subsection{Data processing and analysis}

The data from the three different methods (the GT3X+, the SOPLAY, and the CAM system) were processed to produce comparable PA outcome measures. The observation duration was $20 \mathrm{~min}$ and observations (scans) were made every $2 \mathrm{~min}$. This resulted in 10 observation periods of $10 \mathrm{~s}$ each for each of the eight players. Temporal matching of the data made it possible to match the periods and to directly compare the intensity within the periods across the three methods (GT3X+, CAM, SOPLAY 1 and SOPLAY 2). The percent time spent in three intensity zones (light: $<2 \mathrm{mph}$; walking: $2-4 \mathrm{mph}$; very active: $>4 \mathrm{mph}$ ) were used to provide a comprehensive evaluation of the activity patterns. Because the data were temporally linked it was possible to match the estimates from all three devices in $10 \mathrm{~s}$ epochs. The data from the 
Table 1. Percentage of minutes in PA intensities according to the three instruments.

\begin{tabular}{llll}
\hline PA intensity (Percentage) & Light & Walking & Very active \\
\hline SOPLAY 1 & 39 & 45 & 17 \\
SOPLAY 2 & 27 & 49 & 24 \\
GT3X + & 64 & 18 & 18 \\
CAM $^{\text {a }}$ & 48 & 17 & 34 \\
\hline
\end{tabular}

${ }^{\mathrm{a}}$ GT3X + and CAM use Evenson limits (walking: $2 \mathrm{mph}$, very active: $4 \mathrm{mph}$ ).

Table 2. Results of the Yates Chi-square between instruments.

\begin{tabular}{llllllr}
\hline Yates & & \multicolumn{4}{c}{ Expected } \\
\cline { 1 - 4 } Chi-Square & & & GT3X & SOPLAY 1 & SOPLAY 2 & CAM \\
\hline Observed & GT3X & - & 77.60 & 93.10 & 36.40 \\
& SOPLAY 1 & 119.55 & - & 27.00 & 115.09 \\
& SOPLAY 2 & 144.44 & 46.33 & - & 150.17 \\
& CAM & 24.18 & 110.90 & 158.23 & - \\
\hline
\end{tabular}

accelerometer were first filtered and the specific periods were matched with the corresponding observation scans (i.e. each 10 second periods). The CAM data were calibrated to track and extract the participants' movements with the same cut points used in the accelerometer data (Evenson et al 2008). For example, walking corresponded to intensities of $2 \mathrm{mph}$, while very active periods (running) corresponded to $4 \mathrm{mph}$ or greater. A chi-square test was used to test the differences between the three methods, in the percent times at three intensity zones (light, walking and very active). The Yates' correction was used to prevent the overestimation of statistical significance for small data. All analyses were performed with the Excel software (Microsoft Office Excel 2013, USA) and the level of significance was set at 95\% $(p<0.05)$.

\section{Results}

PA was categorized in percentage of time spent at three levels (light, walking, and very active), and the results are provided in table 1 . The results showed that the practice was predominantly a lower intensity session with the majority of time spent in light activity (according to the GT3+ and CAM). The two observers using the SOPLAY instrument coded a higher percentage of time spent in the walking category during the session.

Chi-square statistics for the difference in PA intensity percentages resulting from the three different instruments (GT3X, SOPLAY and CAM) are provided in table 2. Pairwise comparisons between the GT3X+ and the other methods showed the following significant differences: CAM versus GT3x $+\chi^{2}(5)=36.40, p<.001$; SOPLAY2 versus GT3x+ was $\chi^{2}(5)=93.10$, $p<.001$; SOPLAY1 versus GT3x+ was $\chi^{2}(5)=77.60, p<.001$.

Table 3 presents the mean error (percentage) between the different instruments. The lowest value was between the two SOPLAY observers, SOPLAY 1 versus SOPLAY $2=4.70$. However, this is an indicator of reliability. When the SOPLAY observers were individually compared to the GT3X to evaluate validity, the error was larger (SOPLAY 1 versus GT3X = 8.68; SOPLAY 2 versus GT3X $=9.60$ ) than the corresponding comparisons with the CAM instrument when compared to the GT3X (CAM versus GT3X = 5.32). It is noteworthy that 
Table 3. Percentage mean error between the different instruments.

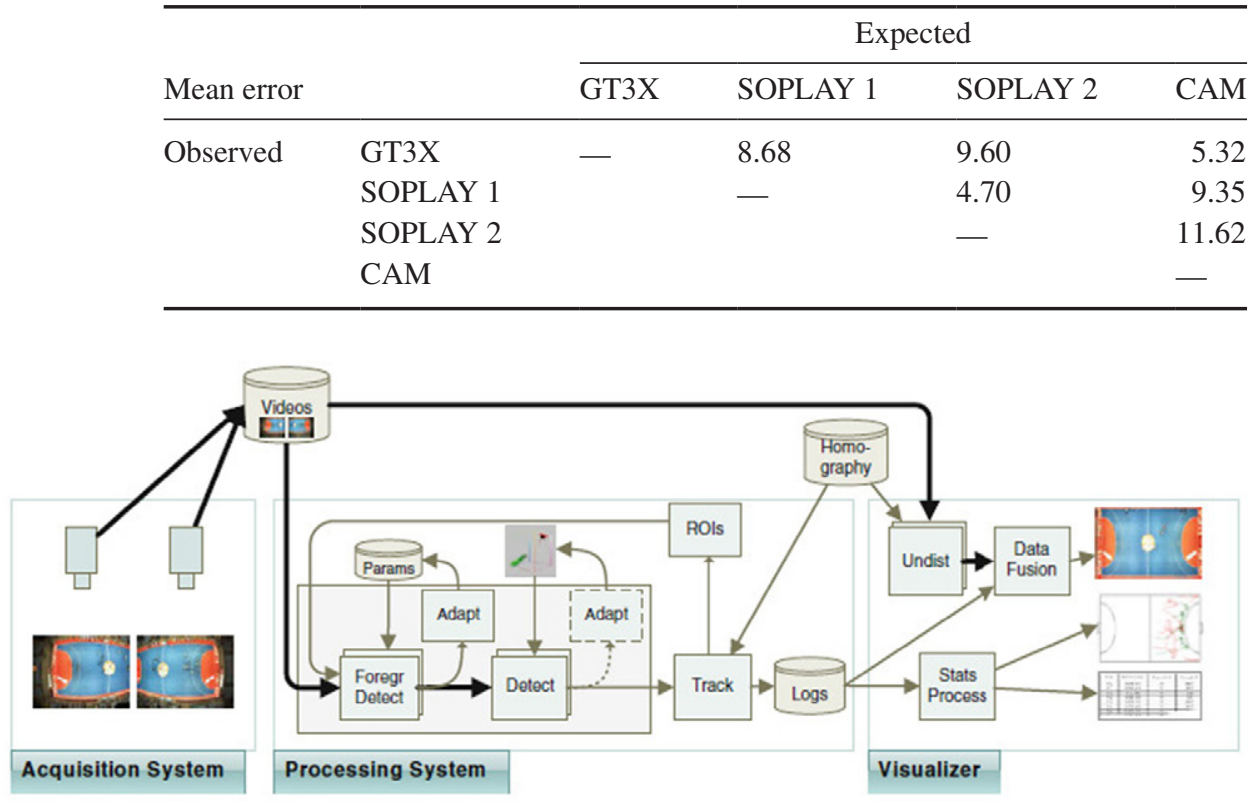

Figure 2. CAM system processing phases, adapted from Santiago et al (2012).

the mean error between the CAM and the GT3X is relatively small, and close to that obtained from the two SOPLAY observers.

\section{Discussion}

The results of this study suggest that the video tracking system (CAM) has the potential to automatically classify PA behavior in the same way as the SOPLAY (three intensity categories: light, walking, and very active). The direct comparison with the GT3X+ shows better agreement with the CAM than with the SOPLAY system, suggesting that this system may have promise. One of the advantages of the CAM system is that (like activity monitors) it can provide continuous monitoring of the behavior. Observation systems such as the SOPLAY only provide $10 \mathrm{~s}$ of behavior classification every few minutes. Another relevant advantage of the CAM system is that it is automated and less intrusive for the participants. It would also present a more cost-effective system since once the capital investment is made the system can be used automatically.

The selection of instruments for measuring PA in a particular study will depend on the type of data that is needed, the study objectives, the characteristics of the target population, as well as feasibility issues such as cost and logistics (Butte et al 2012). In the DO method, there is a trade-off between precision and feasibility. It is desirable to have multiple codes to provide a richer representation of the data but human observers have a limited ability to discriminate among the levels. The requirement to continuously code data over short time periods is burdensome and challenging to sustain over time. These features limit the quality and amount of data that can be reliably captured with standard DO techniques. The SOPLAY is designed to capture random snapshots of activity levels and these snapshots are presumed to reflect the activity behavior of the group during the whole period of time. In our study we 
used 10 s periods of observation for each gender, but how frequent scans have to be made to accurately capture activity levels is still an unresolved question (Saint-Maurice et al 2011b). One of the main advantage of the CAM system over SOPLAY is that it can be trained to track the individuals continually, and depending of the frame rate capabilities of the video camera in use, we can have sampling rates from 30 frames per second to 120 frames per second with a relatively low budget video camera.

Another, important advantage of the CAM system is the elimination of the need to train the observers in DO methods. The accuracy depends on the skills and objectivity of the observer to identify physical activity behaviors so observers must be properly trained to be consistent and nonjudgmental. Steps must also be taken to ensure they maintain their skills over time and there is the possibility of observed people behaving differently when an observer is present (i.e. reactivity). Inter-observer reliability for SOPLAY was previously tested and considered acceptable ( $\mathrm{IOA}=80 \%, R=.75$ ) (McKenzie et al 2000). In this study, observers were trained to try to guarantee adequate data collection, and the smallest measurement error was achieved between the two SOPLAY observers. On the other hand, with the CAM system we can get similar information to the SOPLAY methodology concerning the classification of behavior in three categories (light, walking, and very active), providing evidence that we can substitute it with the semi-automated method, taking away the burden of having two observers. The user intervention required by the CAM system is mostly concerned with selecting the players to be followed and correct player identification during the tracking process, but as the tracking algorithms develop this will no longer be required and the process becomes more automated.

DO methods are underused by researchers because the most significant limitation is the cost and burden associated with collecting and processing data (McKenzie 2010). This barrier typically leads to a limited scope or amount of observation data, bringing into question whether data from those samples are adequate for that setting or whether they can be generalized. These limitations have proven difficult to overcome, but new advances in automated video-based processing techniques offer considerable promise in this area. The future development of sensors and analytical techniques for assessing PA should focus on the dynamic ranges of sensors, comparability for sensor output across manufacturers, and the application of advanced modeling techniques to predict energy expenditure and classify physical activities. New approaches for qualitatively classifying PA should be validated using DO or recording (Butte et al 2012).

With advancements in computer visualization, there will be corresponding improvements in automatically detecting human movements and categorizing behaviors such as PA. Advances in sensor, communications and computer technologies will also clearly continue to advance research to improve PA assessment methodologies (Silva et al 2013). In this study, the CAM system demonstrated promise for characterizing PA behavior. It demonstrated a number of relevant advantages: it wasn't intrusive to the participants (they do not need to wear any device other than a colorful vest); it's independent of the participants' characteristics (such as age, height or weight); it can be programmed to start and stop remotely and automatically; it avoids the burden and the subjectivity of DO methods; it can achieve reasonable sampling rates to collect data (we used 30 frames per second); it can record and track behavior continuously and for prolonged periods of time; it enables re-analyses of the data, allowing the researcher to 'go back in time'; and lastly, it can be fine-tuned and customized to the researcher's needs over time as technology and tracking algorithms improve.

The present study focused on evaluating a single activity (basketball) but it was designed to have utility for other contexts. It uses a camera to retrieve high-quality video, and the system is somewhat portable in sports halls or gyms, which permits its use in different indoor settings such as playing sports, physical education classes or indoor recess periods. The proposed 
methodology was validated with real practice footage filmed at a regular sports session with no constraints. Results show that using simple features such as color combined with a powerful tool for tracking (Kalman Filter), it is possible to detect and track participants throughout the game area with very limited user intervention; initial color calibration by user and little more. The conversion of each player's image coordinates into real-world coordinates (with measurement errors below $35 \mathrm{~cm}$ ) allows the tracking of the players with multiple cameras, but also to extract metrics of the participant's or players' performance (i.e. positions, velocity and distance traveled).

While the system shows promise, we acknowledge that the use of only a single setting and the small sample size are limitations of the study. However, it is important to point out that no constraints were imposed during the practice which permitted the assessment of different PA behaviors normally performed in basketball training (e.g. coach talk, warm-up, skill drills and game play). It is also noteworthy that the CAM system was first used and tested during a professional handball game (Santiago et al 2012) so this study demonstrates applicability in other contexts and indoor settings. Despite its promise, more studies are clearly warranted to verify if it can be applied in more sports or settings such as physical education classes or recess periods.

Surveillance systems must be developed to provide rich, accurate information in a costeffective manner. Video has the advantage of being non-intrusive and enabling more complete contextual characterizations. These new technologies can contribute to a finer temporal resolution of the observations while decreasing the burden on the human observer. They may also enable cross-checking and rectification of coding errors. Moreover, an increase in automation improves the objectivity of the process and extendes the type/diversity of gathered data. These attributes directly overcome some of the stated limitations of current DO tools. With further refinements, this technology can be applied to directly (and automatically) code physical activity behaviors of multiple participants engaging in a group activity.

\section{Acknowledgments}

This work is partially financed by the ERDF-European regional development Fund through the COMPETE Programme (operational programme for competitiveness) and by National Funds through the FCT_-Fundação para a Ciência e a Tecnologia (Portuguese Foundation for Science and Technology) within project 'FCOMP-01-0124-FEDER-037281'.

The authors also wish to thank scholarships SFRH/BPD/71332/2010 and Fundação Calouste Gulbenkian (scholarship with ref. 104410) and funding program PEst-OE/SAU/ UI0617/2011 for the additional partial funding.

\section{References}

Butte N F, Ekelund U and Westerterp K R 2012 Assessing physical activity using wearable monitors: measures of physical activity Med. Sci. Sports Exerc. 44 S5-12

Evenson K R, Catellier D J, Gill K, Ondrak K S and Mcmurray R G 2008 Calibration of two objective measures of physical activity for children J. Sports Sci. 26 1557-65

Hagstromer M, Oja P and Sjostrom M 2007 Physical activity and inactivity in an adult population assessed by accelerometry Med. Sci. Sports Exerc. 39 1502-8

Kristan M, Perš J, Perše M and Kovačič S 2009 Closed-world tracking of multiple interacting targets for indoor-sports applications Comput. Vis. Image Understand. 113 598-611

McKenzie T 2002 Use of direct observation to assess physical activity ed G Welk Physical Activity Assessments for Health-Related Research (Champaign, IL: Human Kinetics) 
McKenzie T L 20102009 C. H. McCloy lecture. Seeing is believing: observing physical activity and its contexts Res. Q. Exerc. Sport. 81 113-22

McKenzie T L, Catellier D J, Conway T, Lytle L A, Grieser M, Webber L A, Pratt C A and Elder J P 2006 Girls' activity levels and lesson contexts in middle school PE: TAAG baseline Med. Sci. Sports Exerc. 38 1229-35

McKenzie T L, Marshall S J, Sallis J F and Conway T L 2000 Leisure-time physical activity in school environments: an observational study using SOPLAY Prev. Med. 30 70-7

Monier E, Wilhelm P and Ruckert U 2009 Template matching based tracking of players in indoor team sports Proc. of the 3rd ACM/IEEE Int. Conf. on Distributed Smart Cameras (ICDSC) (Paderborn, Germany, 2009)

Mota J, Silva P, Aires L, Santos M P, Oliveira J and Ribeiro J C 2008 Differences in school-day patterns of daily physical activity in girls according to level of physical activity J. Phys. Act. Health. $5 \mathrm{~S} 90-7$

Needham C and Boyle R 2001 Tracking multiple sports players through occlusion, congestion and scale Proc. of the British Machine Vision Conf. (Manchester, UK, 2001) pp 93-102

Pratt M, Sarmiento O L, Montes F, Ogilvie D, Marcus B H, Perez L G and Brownson R C 2012 The implications of megatrends in information and communication technology and transportation for changes in global physical activity Lancet $380282-93$

Riddoch C J, Bo Andersen L, Wedderkopp N, Harro M, Klasson-heggebo L, Sardinha L B, Cooper A R and Ekelund U 2004 Physical activity levels and patterns of 9- and 15 year-old European children Med. Sci. Sports Exerc. 36 86-92

Saint-Maurice P F, Welk G, Ihmels M A and Krapfl J R 2011a Validation of the SOPLAY direct observation tool with an accelerometry-based physical activity monitor J. Phys. Act. Health $81108-16$

Saint-Maurice P F, Welk G J, Silva P, Siahpush M and Huberty J 2011b Assessing children's physical activity behaviors at recess: a multi-method approach Pediatr. Exerc. Sci. 23 585-99

Santiago C, Sousa A and Reis L 2012 Vision system for tracking handball players using fuzzy color processing Mach. Vis. Appl. 24 1055-74

Silva P, Andrade M T, Carvalho P and Mota J 2013 A structured and flexible language for physical activity assessment and characterization J. Sports Med. 20139

Silva P, Santos R, Welk G and Mota J 2011 Seasonal differences in physical activity and sedentary patterns: the relevance of the PA context J. Sports Sci. Med. 10 66-72

Troiano R P, Berrigan D, Dodd K W, Masse L C, Tilert T and Mcdowell M 2008 Physical activity in the United States measured by accelerometer Med. Sci. Sports Exerc. 40 181-8

Trost S G, Pate R R, Freedson P S, Sallis J F and Taylor W C 2000 Using objective physical activity measures with youth: how many days of monitoring are needed? Med. Sci. Sports Exerc. 32 426-31 